\title{
Correction to: \\ Some Oscillation and Asymptotic Properties for Linear Differential Equations
}

(Bull. Fac. Sci., Ibaraki Univ., Math., No. 8, 1976, pp. 25-30)

\section{A. Staikos* and Ch. G. Philos*}

The purpose of this note is to present some radical changes on the results of our paper mentioned above. These changes are necessary due to the fact that most of the results of our paper have been obtained following an erroneous step based on a similar argument by Singh [1]. The authors are indebted to Prof. H. Onose for his remarks on the subject. The above step could be easily modified in order that the results hold as exactly as they are formulated in the case of second order differential equations, while they hold at least for the bounded solutions in the case of differential equations of arbitrary order. This could be done by choosing the points $t_{1}, \tau_{1}, \tau_{2}, \ldots, \tau_{n-1}$ (line 1 from below, p. 26 and line 10 from below, p. 28) so that $T<t_{1}<\tau_{1}<\tau_{2}<\cdots<\tau_{n-1}$.

We present here the corrected results and provide a new proof for the theorems which is a lot more simple.

We consider the $n$-th order $(n>1)$ general ordinary differential equation

$$
\left(r_{n-1}(t)\left(r_{n-2}(t)\left(\cdots\left(r_{1}(t)\left(r_{0}(t) x(t)\right)^{\prime}\right)^{\prime} \cdots\right)^{\prime}\right)^{\prime}\right)^{\prime}+a(t) x(t)=b(t), \quad t \geqq t_{0},
$$

where the functions $r_{i}(i=0,1, \ldots, n-1)$ are positive at least on the interval $\left[t_{0}, \infty\right)$. The continuity of the functions $r_{i}(i=0,1, \ldots, n-1), a$ and $b$ will be assumed without mention. Because of the linearity, all solutions of (E) are defined on the whole interval $\left[t_{0}, \infty\right)$. The oscillatory character is considered in the usual sense, i.e. a continuous real-valued function which is defined on an interval of the form $[T$, $\infty)$ is called oscillatory if it has no last zero, and otherwise it is called nonoscillatory.

THEOREM 1. Consider the differential equation (E) subject to the conditions:

$$
\liminf _{t \rightarrow \infty} r_{0}(t)>0 \text {. }
$$

(A) $\int^{\infty} \frac{1}{r_{1}\left(s_{1}\right)} \int_{s_{1}}^{\infty} \frac{1}{r_{2}\left(s_{2}\right)} \cdots \int_{s_{n-2}}^{\infty} \frac{1}{r_{n-1}\left(s_{n-1}\right)} \int_{s_{n-1}}^{\infty}|a(s)| d s d s_{n-1} \cdots d s_{2} d s_{1}<\infty$.

Received June 2, 1978

* Department of Mathematics, University of Ioannina, Ioannina, G reece. 
(B) $\quad \int^{\infty} \frac{1}{r_{1}\left(s_{1}\right)} \int_{s_{1}}^{\infty} \frac{1}{r_{2}\left(s_{2}\right)} \cdots \int_{s_{n-2}}^{\infty} \frac{1}{r_{n-1}\left(s_{n-1}\right)} \int_{s_{n-1}}^{\infty}|b(s)| d s d s_{n-1} \cdots d s_{2} d s_{1}<\infty$.

Then for every bounded oscillatory solution $x$ of the equation (E),

$$
\lim _{t \rightarrow \infty} x(t)=0 \text {. }
$$

Moreover, in the particular case $n=2$ all unbounded solutions of (E) are nonoscillatory.

Theorem 2. Consider the differential equation

$$
\left(r_{n-1}(t)\left(r_{n-2}(t)\left(\cdots\left(r_{1}(t)\left(r_{0}(t) x(t)\right)^{\prime}\right)^{\prime} \cdots\right)^{\prime}\right)^{\prime}\right)^{\prime}+a(t) x(t)=0
$$

subject to the conditions $\left(\mathrm{R}_{0}\right)$ and $(\mathrm{A})$.

Then every nontrivial bounded solution of the equation $(\mathrm{E})_{0}$ is nonoscillatory.

Proofs of Theorems 1 and 2. Let $x$ be an oscillatory solution of (E). We introduce the notations $D_{r}^{(i)} x(i=0,1, \ldots, n-1)$ as follows:

$$
D_{r}^{(0)} x=r_{0} x \quad \text { and } \quad D_{r}^{(i)} x=r_{i}\left(D_{r}^{(i-1)} x\right)^{\prime} \quad(i=1,2, \ldots, n-1) .
$$

Obviously, all functions $D_{r}^{(i)} x(i=0,1, \ldots, n-1)$ are also oscillatory. We put $c=\inf \left\{r_{0}(t): t \geqq t_{0}\right\}$. Because of $\left(\mathrm{R}_{0}\right)$, we have that $c>0$.

Let $x$ be bounded. For any fixed $t \geqq t_{0}$ we choose $\tau_{i}(i=0,1, \ldots, n-1)$ with $t \leqq \tau_{0} \leqq \tau_{1} \leqq \cdots \leqq \tau_{n-1}$ and $\left(D_{r}^{(i)} x\right)\left(\tau_{i}\right)=0(i=0,1, \ldots, n-1)$. Then on repeated integration from equation (E) we obtain

$$
\begin{array}{r}
(-1)^{n}\left(D_{r}^{(0)} x\right)(t)+\int_{t}^{\tau_{0}} \frac{1}{r_{1}\left(s_{1}\right)} \int_{s_{1}}^{\tau_{1}} \frac{1}{r_{2}\left(s_{2}\right)} \cdots \int_{s_{n-2}}^{\tau_{n-2}} \frac{1}{r_{n-1}\left(s_{n-1}\right)} \int_{s_{n-1}}^{\tau_{n-1}} a(s) x(s) d s d s_{n-1} \cdots \\
\cdots d s_{2} d s_{1} \\
=\int_{t}^{\tau_{0}} \frac{1}{r_{1}\left(s_{1}\right)} \int_{s_{1}}^{\tau_{1}} \frac{1}{r_{2}\left(s_{2}\right)} \cdots \int_{s_{n-2}}^{\tau_{n-2}} \frac{1}{r_{n-1}\left(s_{n-1}\right)} \int_{s_{n-1}}^{\tau_{n-1}} b(s) d s d s_{n-1} \cdots \\
\cdots d s_{2} d s_{1} .
\end{array}
$$

Therefore, for every $t \geqq t_{0}$ we have

(*) $|x(t)| \leqq \frac{S}{c} \int_{t}^{\infty} \frac{1}{r_{1}\left(s_{1}\right)} \int_{s_{1}}^{\infty} \frac{1}{r_{2}\left(s_{2}\right)} \cdots \int_{s_{n-2}}^{\infty} \frac{1}{r_{n-1}\left(s_{n-1}\right)} \int_{s_{n-1}}^{\infty}|a(s)| d s d s_{n-1} \cdots$

$$
\cdots d s_{2} d s_{1}
$$

$$
+\int^{\infty} \frac{1}{r_{1}\left(s_{1}\right)} \int_{s_{1}}^{\infty} \frac{1}{r_{2}\left(s_{2}\right)} \cdots \int_{s_{n-2}}^{\infty} \frac{1}{r_{n-1}\left(s_{n-1}\right)} \int_{s_{n-1}}^{\infty}|b(s)| d s d s_{n-1} \ldots
$$

$\cdots d s_{2} d s_{1}$,

where $S=\sup \left\{|x(t)|: t \geqq t_{0}\right\}$. Now, by conditions (A) and (B), inequality (*) gives

$$
\lim _{t \rightarrow \infty} x(t)=0
$$


Next, we assume that $b=0$. If $x$ is in addition nontrivial, then from (*) we get

$$
0<c \leqq \int_{1}^{\infty} \frac{1}{r_{1}\left(s_{1}\right)} \int_{s_{1}}^{\infty} \frac{1}{r_{2}\left(s_{2}\right)} \cdots \int_{s_{n-2}}^{\infty} \frac{1}{r_{n-1}\left(s_{n-1}\right)} \int_{s_{n-1}}^{\infty}|a(s)| d s d s_{n-1} \cdots d s_{2} d s_{1},
$$

which contradicts condition (A).

It remains to prove the second part of the conclusion of Theorem 1. This is the case of $n=2$. Because of (A) and (B), we choose a $T \geqq t_{0}$ so that

$$
\int_{T}^{\infty} \frac{1}{r_{1}(t)} \int_{t}^{\infty}|a(s)| d s d t \leqq \frac{c}{2} \text { and } \int_{T}^{\infty} \frac{1}{r_{1}(t)} \int_{t}^{\infty}|b(s)| d s d t \leqq \frac{c}{2}
$$

and we consider a $\tau_{0} \geqq T$ with $x\left(\tau_{0}\right)=0$. Next, we suppose that $x$ is unbounded and let $\hat{\tau}_{0}>\tau_{0}$ be such that $\left|x\left(\hat{\tau}_{0}\right)\right|>1$. Also, we choose a $\tau_{1} \geqq \hat{\tau}_{0}$ with $\left(D_{r}^{(1)} x\right)\left(\tau_{1}\right)=0$. Then from equation (E) we obtain

$$
\left(D_{r}^{(0)} x\right)(t)-\int_{\tau_{0}}^{t} \frac{1}{r_{1}(u)} \int_{u}^{\tau_{1}} a(s) x(s) d s d u=-\int_{\tau_{0}}^{t} \frac{1}{r_{1}(u)} \int_{u}^{\tau_{1}} b(s) d s d u
$$

and consequently for every $t \in\left[\tau_{0}, \tau_{1}\right]$ we get

$$
\begin{aligned}
\left|\left(D_{r}^{(0)} x\right)(t)\right| & \leqq \int_{\tau_{0}}^{t} \frac{1}{r_{1}(u)} \int_{u}^{\tau_{1}}|a(s)||x(s)| d s d u+\int_{\tau_{0}}^{t} \frac{1}{r_{1}(u)} \int_{u}^{\tau_{1}}|b(s)| d s d u \\
& \leqq M \int_{T}^{\infty} \frac{1}{r_{1}(u)} \int_{u}^{\infty}|a(s)| d s d u+\int_{T}^{\infty} \frac{1}{r_{1}(u)} \int_{u}^{\infty}|b(s)| d s d u \\
& \leqq M \frac{c}{2}+\frac{c}{2}
\end{aligned}
$$

where $M=\max \left\{|x(t)|: t \in\left[\tau_{0}, \tau_{1}\right]\right\}$. Thus, $M \leqq \frac{1}{2} M+\frac{1}{2}$, i.e. $M \leqq 1$, which is a contradiction, since $M \equiv \max \left\{|x(t)|: t \in\left[\tau_{0}, \tau_{1}\right]\right\} \geqq\left|x\left(\hat{\imath}_{0}\right)\right|>1$.

\section{References}

[1] Bhagat Singh, Forced oscillations in general ordinary differential equations, Tamkang J. Math. 6 (1975), 5-11. 